\title{
Model Studies of the Interfacial Ordering of Oleanolic Acid in the Cuticula
}

\author{
Gerald Brezesinski and Dieter Vollhardt*
}

((Dedication----optional))

Triterpenoids represent a promising and expanding platform for biologically active natural compounds whose capability to unique and potentially usable biological effects is currently only partly utilized. They are present in a variety of common plants and fruits ${ }^{[1]}$ and the triterpenoid isolation and identification from such natural sources is of special interest. ${ }^{[2]}$ From the biological standpoint, oleane triterpenoids belong to the most important triterpenoid structures. Besides the continuing extraction and isolation of natural material, synthetic derivatives with higher therapeutic potential have been developed. ${ }^{[1,3]}$ Oleanolic acid is an omnipresent triterpenoid in plant kingdom, medical herbs and is integral part of the human diet. It is one of the most important components of the cuticular membrane of plant leaves and fruits. Important functions for the performance of the cuticular membrane, such as protection from diseases, insects and drought, are related to the composition and molecular arrangement of the epicuticular waxes. ${ }^{[4,5]}$ Recent pharmacological activities on the basis of oleanolic acid comprised the isolation and purification of oleanolic acid from various plants and herbs, the chemical modifications to create more effective derivatives, the toxicity studies and the clinical use in various diseases including anticancer chemotherapies. ${ }^{[3]}$

The omnipresence of oleanolic acid in epicuticular waxes suggests its important role for the cuticle performance. Though nature and chemical composition of the epicuticular waxes are variable, most of the cuticle components are amphiphiles so that a layered structure of the cuticle morphology can be expected. However, the chemical structure of oleanolic acid with a multicyclic planar structure deviates from the typical structure of amphiphiles consisting of a polar head group and alkyl chain(s). Despite the untypical amphiphilic character of oleanolic acid, it is located exclusively in the cuticula. Therefore, studies of well-defined model monolayers at the air $/ \mathrm{w}^{\mathrm{i}}$ ater interface are a promising approach for getting first insight into the specific behaviour of oleanolic acid. A recent paper about mixed oleanolic acid/stearic acid monolayers could not provide convincing information about the structural ordering of pure oleanolic monolayers. ${ }^{[6]}$ Therefore the present work focuses on the interfacial ordering of pure oleanolic acid monolayers. Grazing incidence X-ray diffraction (GIXD) and specular X-ray reflectivity (SR) measurements have proven to be optimal on the basis of lateral pressure results.

First information about the features of the oleanolic acid spread

[*] PD Dr. G. Brezesinski, Prof. Dr. D. Vollhardt Department of Interfaces

Max Planck Institute of Colloids and Interfaces

Research Campus Golm, 14476 Potsdam (Germany)

Fax: (+49) 331-567-9202

E-mail: dieter.vollhardt@mpikg.mpg.de

[**] The authors thank PD Dr. H. Cölfen for the structure calculations of oleanolic acid. on pure water at $25^{\circ} \mathrm{C}$ can be obtained from $\pi$-A isotherm shown in Fig. 1. The chemical structure in the inset of Fig. 1 indicates the untypical amphiphilic character of the bulky triterpenoid oleanolic acid. It is seen that a correct $\pi$-A isotherm for oleanolic acid monolayers can be measured only up to $\pi \sim 8 \mathrm{mN} \cdot \mathrm{m}^{-1}$. In the $0 \leq \pi \leq$ $8 \mathrm{mN} \cdot \mathrm{m}^{-1}$ range, repeated compression and expansion confirmed the measured shape of the isotherm. This result shows unambiguously that oleanolic acid forms insoluble monolayers. However, the extreme stiffness of the compressed monolayers prevents artifactfree recording of $\pi-\mathrm{A}$ isotherms up to high lateral pressures so that at $\pi>8 \mathrm{mN} \cdot \mathrm{m}^{-1}$ the recorded $\pi$ values deviate increasingly from the expected real value.

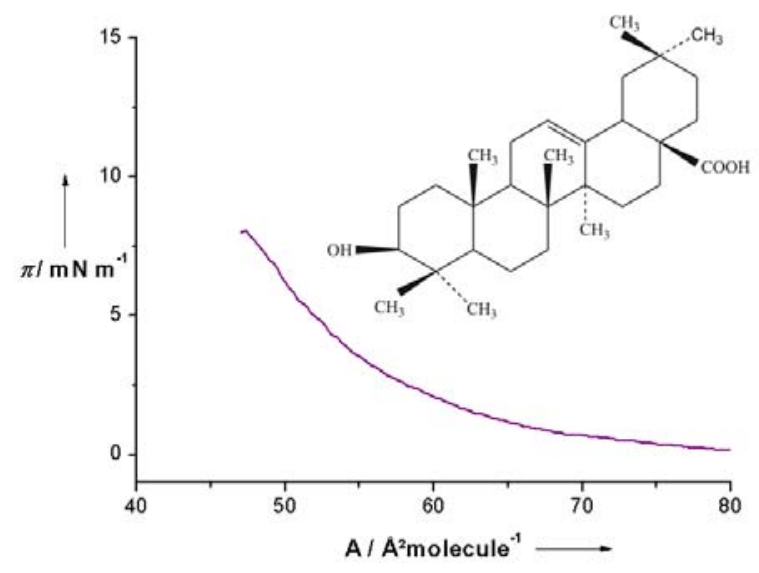

Figure 1. $\pi$-A isotherms of oleanolic acid monolayer spread on pure water at $25^{\circ} \mathrm{C}$. The inset shows the chemical structure of oleanolic acid.

Reliable knowledge of the characteristics of oleanolic acid monolayers are possible on the basis of direct structural information. Grazing incidence X-ray diffraction (GIXD) gives information about the two-dimensional symmetry of monolayers on the $\AA$-scale. GIXD is sensitive to the condensed parts of the monolayer, whereas the unordered liquid-expanded phase contributes only to the background scattering.

All diffraction studies were performed on water at different lateral pressures at $25^{\circ} \mathrm{C}$. Figure 2 shows the corrected X-ray intensities as a function of the in-plane scattering vector component $Q_{x y}$ (Bragg peak, above) and in the corresponding contour plot as a function of the in-plane and out-of-plane scattering vector components $Q_{x y}$ and $Q_{z}$ (bottom left) for the oleanolic acid monolayer in the compressed state (approximately $8 \mathrm{mN} \cdot \mathrm{m}^{-1}$ ). Only one Bragg peak can be observed, just as at all accessible lateral pressures. Integration over the whole $\mathrm{Q}_{\mathrm{z}}$-window of the detector and fitting a Lorentzian function to the experimental data show that this 
peak is located at a $Q_{x y}$-value of $1.017 \AA^{-1}$. The position of this Bragg peak describes a hexagonal packing of the oleanolic acid molecules in the monolayers (LS phase) with a d value of $6.178 \AA$ leading to a cross-section area of $44.1 \AA^{2} \cdot$ molecule $^{-1}$. This is in good agreement with the estimated value of the cross-section area of 44.2 $\AA^{2} \cdot$ molecule $^{-1}$ using oleanolic acid molecular data and assuming free rotation around the long molecular axis with a diameter of $7.5 \AA$ of the cylindrical molecule. The thickness $L_{z}$ of the oleanolic acid layer is of further interest to characterize the two-dimensional packing features of the monolayer. The thickness of the diffracting layer can be estimated from the full-width at half maximum (fwhm) of the Bragg rod (Fig. 2, bottom right) using $L_{z} \sim(0.9 \cdot 2 \pi) / \mathrm{fwhm}\left(Q_{z}\right)$. The thickness $L_{z}$ of the oleanolic acid monolayer amounts to $14.9 \AA$ resulting from the average value of the $\operatorname{fwhm}\left(Q_{z}\right)$ of $0.38 \AA^{-1}$.
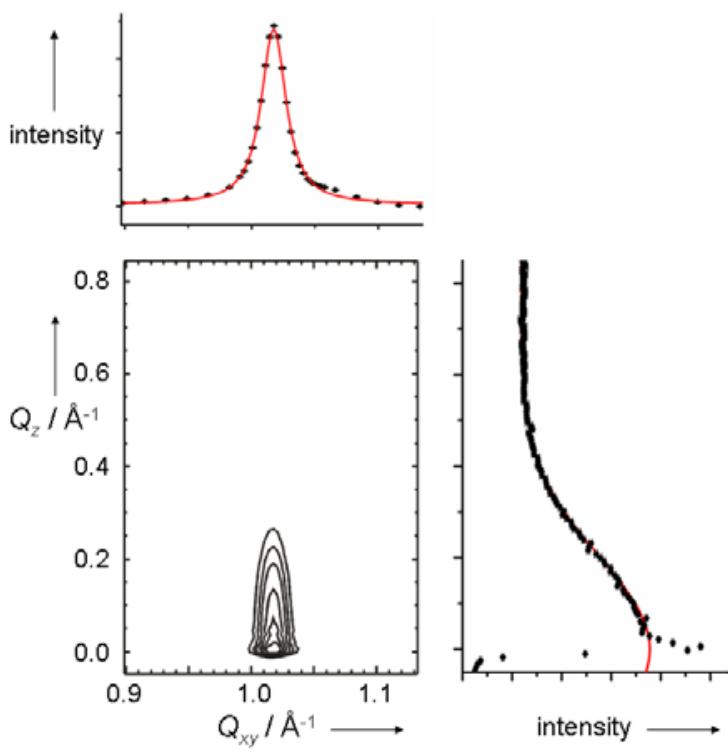

Figure 2. Grazing incidence X-ray diffraction data for an oleanolic acid monolayer spread on water at $25^{\circ} \mathrm{C}$ and compressed to $8 \mathrm{mN} \cdot \mathrm{m}^{-1}$. The diffracted intensity, corrected for polarization, effective area, and Lorentz factor, is plotted as contour lines of equal intensity versus the in-plane component $Q_{x y}$ and the out-of-plane component $Q_{z}$ of the scattering vector (bottom left). Integration of the measured intensity over a $Q_{z}$ range from 0 to $0.5 \AA^{-1}$ yields a single Bragg peak (top), whereas integration over a $Q_{x y}$ range from $0.9 \AA^{-1}$ to $1.14 \AA^{-1}$ yields the Bragg rod (bottom right), which is superimposed by the sharp Yoneda-Vineyard peak. The horizontal resolution of $0.008 \AA^{-1}$ was determined by a Soller collimator mounted in front of the PSD. Model peaks (solid lines) taken as Lorentzian in the in-plane direction and as Gaussian in the out-of-plane direction were least-square fitted to the measured intensities.

Usually the monolayer consists of 2D crystallites that are perfect and have a finite size $L_{x y}$. This size can be estimated by $L_{x y} \sim$ $(0.9 \cdot 2 \pi) / f w h m\left(Q_{x y}\right)$. Similar to the exponential decay of the correlation, as found in liquid crystals, the fwhm $\left(Q_{x y}\right)$ corresponds to the correlation length $\xi=2 / f w h m\left(Q_{x y}\right)$. The measured fwhm corrected by the instrumental resolution leads to a size of the $2 \mathrm{D}$ crystallites $L_{x y} \approx 260 \AA(\xi \approx 93 \AA)$ in the observed LS phase. This value is considerably smaller compared with those of typical wellpacked amphiphilic monolayers consisting of alkyl chain(s) and a polar headgroup, such as fatty acids.

To confirm the structural characteristics obtained by GIXD, the thickness of oleanolic acid monolayers was additionally derived from specular X-ray reflectivity (XR) data. Figure 3 (top) shows the reflectivity curve for the oleanolic acid monolayer at $8 \mathrm{mN} \cdot \mathrm{m}^{-1}$ normalized to the Fresnel reflectivity. The comparison of the electron density profile (Fig. 3 bottom) obtained from the inversion of the $\mathrm{R} / \mathrm{R}_{\mathrm{F}}$ using a model-independent approach with that of the bare water surface shows distinct differences. Describing this electron density profile by a one-box model and using the molecular area determined from the $\pi-\mathrm{A}$ isotherm and the number of electrons of the oleanolic acid molecule as fixed parameters gives a box thickness of $z=(15.2 \pm 0.4) \AA$ with an average electron density of $\rho$ $=0.332$ electrons $\cdot \AA^{-3}\left(\rho / \rho_{\text {water }}=0.993\right)$. Thus, the thicknesses of the oleanolic acid monolayer determined by GIXD and XR coincide perfectly, what is surprising considering the bad contrast of the oleanolic acid layer compared with the electron density of water. The obviously tight packing of the perpendicularly oriented oleanolic acid molecules at the water surface leads to a roughness of the layer $(2.9 \AA)$ which is not larger than the roughness of water.
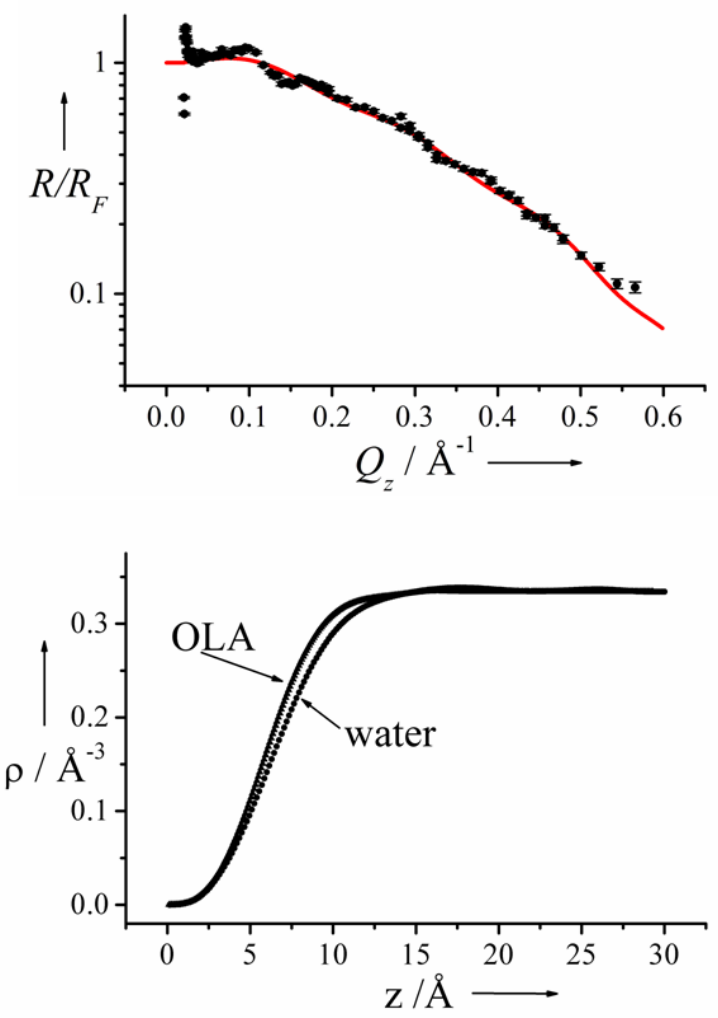

Figure 3. Top: Specular X-ray reflectivity normalized by the Fresnel reflectivity, $\mathrm{R}\left(Q_{z}\right) / \mathrm{R}_{\mathrm{F}}\left(Q_{z}\right)$, for a water surface covered with an oleanolic acid monolayer at $8 \mathrm{mN} \cdot \mathrm{m}^{-1}$. The solid line represents the calculated reflectivity using a model-independent approach. Bottom: Electron density profile $\rho(z)$ versus the depth $z$ corresponding to the calculated reflectivity of the oleanolic acid (OLA) monolayer shown above. The electron density profile of the bare water surface is shown for comparison.

Fig. 4 illustrates schematically the packing of oleanolic acid molecules in a monolayer at the air/water interface in a side view calculated using Cerius ${ }^{2}$ Vers. 4.6. (Accelrys). ${ }^{[7]}$ Both GIXD and $\mathrm{XR}$ results are consistent with the presented perpendicular orientation of the oleanolic acid molecules in a rotator phase (LS) in the whole lateral pressure region. The molecule contains two hydrophilic groups $(\mathrm{COOH}$ and $\mathrm{OH})$ in opposite positions. According to the GIXD and XR results, the ordering with perpendicular arrangement of the molecules presented in Fig. 4 is 
most probable. The $\mathrm{COOH}$ group is directed towards the aqueous subphase because of its higher polarity and more options to form hydrogen bonds, and, consequently, the less polar $\mathrm{OH}$ group must be exposed to the hydrophobic air. This energetically unfavorable direction of the $\mathrm{OH}$ groups is obviously compensated by strong lateral hydrophobic interactions between the tightly packed molecules.

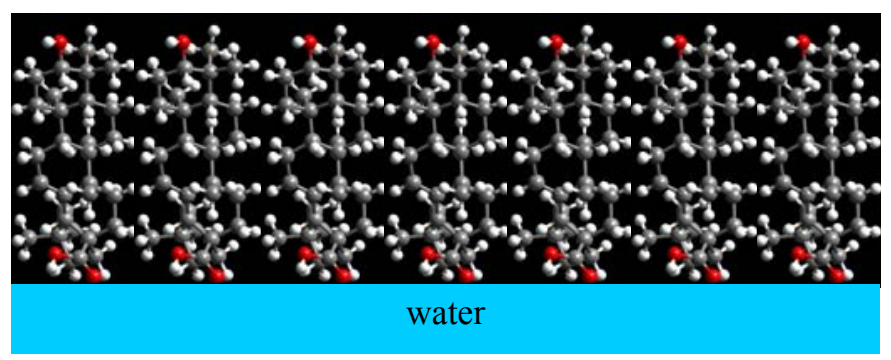

Figure 4. Schematic illustration of the upright orientation of oleanolic acid molecules in a monolayer on water at $10 \mathrm{mN} \cdot \mathrm{m}^{-1}$. The $\mathrm{COOH}$ group is directed towards the aqueous subphase due to its higher polarity and more options to form hydrogen bonds. The energetically unfavorable orientation of the $\mathrm{OH}$ group is compensated by hydrophobic interactions in the tightly packed monolayer.

\section{Experimental Section}

Oleanolic acid, purchased from Sigma in a nominal $\geq 99 \%$ purity, was used without further purification. Ultrapure deionized water with a conductivity of $0.055 \mu \mathrm{S} / \mathrm{cm}$ and $\mathrm{pH} 5.8$ produced by the "Purelab Plus" system (Seral, Germany) was used for the monolayer experiments. The spreading solvent was chloroform (p.a. grade, Baker, Holland).

The surface pressure studies were performed in a self-made computerinterfaced film balance equipped with a Wilhelmy balance for the surface pressure determination and a temperature control system. ${ }^{[8]}$

The lateral structure in the condensed oleanolic acid monolayer was investigated using grazing incidence X-ray diffraction measurements at the BW1 beamline, HASYLAB, DESY (Hamburg, Germany). A Langmuir film balance equipped with a single movable barrier and a Wilhelmy plate for monitoring the lateral pressure was placed in a hermetically closed container filled with helium. At BW1, a monochromatic synchrotron X-ray beam $(\lambda=$ $1.304 \AA$ ) was adjusted to strike the helium/water interface at a grazing incidence angle $\alpha_{i}=0.85 \alpha_{\mathrm{c}}\left(\alpha_{c}=0.13^{\circ}\right)$ and illuminated roughly $2 \times 50 \mathrm{~mm}^{2}$ of the surface. During the measurements, the trough was laterally moved to avoid any sample damage by the strong X-ray beam. A linear positionsensitive detector (PSD, OEM-100-M, Braun, Garching, Germany) was rotated to scan the in-plane $Q_{x y}$ component values of the scattering vector. The vertical channels of the PSD measured the out-of-plane $Q_{z}$ component of the scattering vector between 0 and $1 \AA^{-1}$. The diffraction data consisted of Bragg peaks at diagnostic $Q_{x y}$ values. The in-plane lattice repeat distances $d$ of the ordered monolayer structure were calculated from the Bragg peak positions: $d=2 \pi / Q_{x y}$. The in-plane coherence length $L_{x y}$, was approximated from the full-width at half maximum (fwhm) of the Bragg peak using $L_{x y}$ $0.9(2 \pi) / \mathrm{fwhm}\left(Q_{x y}\right)$. The diffracted intensity normal to the interface was integrated over the $Q_{x y}$ window of the diffraction peak to calculate the corresponding Bragg rod. The thickness of the monolayer was estimated from the fwhm of the Bragg rod using $0.9 \cdot 2 \pi /$ fwhm $\left(Q_{z}\right)$. Experimental details are described in the literature. ${ }^{[9-14]}$

Specular X-ray reflectivity (XR) experiments were performed using the same liquid-surface diffractometer on the undulator beamline BW1. The experimental setup and evaluation procedure have been described in detail elsewhere. ${ }^{[15-17]}$ XR experiments reveal information on the electron-density distribution along the surface normal and can be used to determine the thickness of thin layers. The reflected intensity was measured by a NaI scintillation detector as a function of the vertical incidence angle, $\alpha_{\mathrm{i}}$, with the geometry $\alpha_{i}=\alpha_{f}=\alpha$, where $\alpha_{f}$ is the vertical exit angle of the reflected Xrays. The vertical scattering vector component $Q_{z}=(4 \pi / \lambda) \sin \left(\alpha_{\mathrm{f}}\right)$ was measured in a range between $0.01-0.85 \AA^{-1}$. The background scattering from, e.g., the subphase was measured at $2 \theta_{\text {hor }}=0.7^{\circ}$ and subtracted from the signal measured at $2 \theta_{\text {hor }}=0^{\circ} .{ }^{[15-17]}$ The reflectivity data were inverted by applying a model-independent approach. ${ }^{[18]}$ The obtained electron density profile was interpreted by applying either a box model or assuming a symmetrical electron density distribution.

Received: ((will be filled in by the editorial staff)) Published online on ((will be filled in by the editorial staff))

Keywords: amphiphiles $\cdot$ GIXD $\cdot$ monolayers $\cdot$ oleanolic acid $\cdot$ structure

[1] P. Dzubak, M. Hajduch, D. Vydra, A. Hustova, M. Kvasnica, D. Biedermann, L. Markova,M. Urban, J. Sarek, Nat. Prod. Rep. 2006, 23, 394-411.

[2] J. D. Connolly, R. A. Hill, Nat. Prod. Rep. 2005, 22, 230-248 and 2005, 22, 487-503.

[3] J. Liu, J. Ethnopharmacology 2005, 100, 92-94.

[4] C. G. Casado, A. Heredia, Biochim. Biophys. Acta 2001, 1511, 291296.

[5] A. Heredia, Biochim. Biophys. Acta 2003, 1620, 1-7.

[6] A. C. T. Teixeira, A. C. Fernandes, A. R. Garcia, L. M. Ilharco, P. Brogueira, A. M. P. S. Goncalves da Silva, Chem. Phys. Lipids 2007, 149, 1-13.

[7] A. K. Rappe, C.J. Casewit, K. S. Colwell, W. A. Goddard, W. M. Skiff, J. Am. Chem. Soc. 1992, 11, 10024-10035.

[8] D. Vollhardt, Adv. Colloid Interface Sci. 1996, 64, 143-171.

[9] V. M. Kaganer, I. R. Peterson, R. M. Kenn, M. C. Shih, M. Durbin, P. Dutta, J. Chem. Phys. 1995, 102, 9412-9422.

[10] D. Jacquemain, F. Leveiller, S. Weisbuch, M. Lahav, L. Leiserowitz, K. Kjaer, J. Als-Nielsen, J. Am. Chem. Soc. 1991, 113, 7684-7691.

[11] J. Als-Nielsen, D. Jaquemain, K. Kjaer, M. Lahav, F. Levellier, L. Leiserowitz, Phys. Rep. 1994, 246, 251-313.

[12] K. Kjaer, Physica B 1994, 198, 100-109.

[13] G. Brezesinski, A. Dietrich, B. Struth, C. Böhm, W. G. Bouwman, K. Kjaer, H. Möhwald, Chem. Phys. Lipids 1995, 76, 145-157.

[14] V. M. Kaganer, H. Möhwald, P. Dutta, Rev. Mod. Phys. 1999, 71, 779-819.

[15] C. A. Helm, H. Möhwald, K. Kjaer, J. Als-Nielsen, Eur. Lett. 1987, 4, 697.

[16] M. Schalke, P. Krüger, M. Weygand, M. Lösche, Biochim. Biophys. Acta B 2000, 1464, 113-126.

[17] T. R. M. Jensen, K. Kjaer in Novel Methods To Study Interfacial Layers, Studies in Interface Science, Vol. 11 (Eds.: D. Moebius, R. Miller), Elsevier Science, New York, 2001; pp 205-254.

[18] J. S. Pedersen, I. W.Hamley, J. Appl. Crystallogr. 1994, 27, 36-49. 


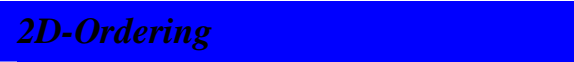

Gerald Brezesinski, Dieter Vollhardt* Page - Page

Model Studies of the Interfacial Ordering of Oleanolic Acid in the Cuticula

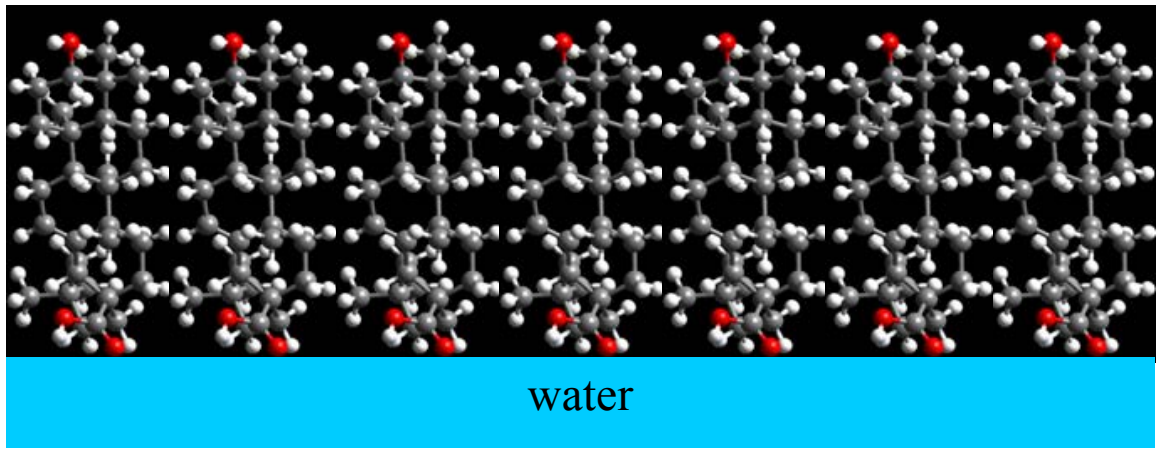

The specific interfacial ordering and structural characteristics of well-defined model monolayers, obtained with highly sophisticated methods, allow first understanding of the oleanolic acid's location exclusively in the cuticula although its chemical multicyclic planar structure deviates considerably from the typical amphiphile's structure. 